\title{
CULTURE ET POLITIQUE À LA RENAISSANCE
}

Terence CAve, Pré-histoires. Textes troublés au seuil de la modernité. Genève, Droz, 1999. $15 \times 22$, 200 p., postf., bibliogr., index (Les Seuils de la modernité, vol. I, Cahiers d'humanisme et Renaissance, 54).

Dans cet important ouvrage qui suit de près la traduction française de Cornucopia, Terence Cave revient sur des problèmes auxquels il a consacré plusieurs articles ces dix dernières années en renouvelant son approche par des investigations inédites. De l'aveu même de l'auteur, le but d'un tel approfondissement n'est pas d'apporter un supplément d'érudition, ni de chercher à tracer des courants à la faveur d'un rapprochement de sources. En bref, Pré-histoires n'est pas une contribution traditionnelle à l'histoire des idées; les textes abordés ne sont convoqués qu'afin d'éprouver la pertinence d'une méthode tout en la précisant. En effet, l'objectif que se donne cet ouvrage est d'ordre méthodologique.

La démarche revendiquée par Cave exige l'établissement d'une configuration «préhistorique » avant toute ambition de parvenir à un discours « historique ». Ce dernier écrase trop souvent la singularité irréductible des textes sous des perspectives téléologiques établies à partir d'une démarche fondée sur l'analepse. Cave y oppose un principe de précaution fondamental : ne pas subsumer les textes sous une histoire préétablie où leur valeur historique est dissoute au sein d'une ligne interprétative trop globale, refuser toute articulation a priori entre eux, «morceler les éléments d'un récit trop rassurant» et les inscrire dans un «champ virtuel pour lequel l'avenir que nous connaissons n'a pas encore eu lieu », champ des possibles qu'il faut, à contre-courant des pratiques ordinaires en histoire des idées, reconquérir par une véritable suspension du jugement méthodologique; il s'agirait ainsi de retrouver, sous des objets historicisés, des fragments d'expérience ouverts sur l'inconnu et dont le sens se fixe dans l'écriture. Il faudrait donc aborder le «moi » montaignien sans que Pascal ou Descartes n'anime les Essais d'un mouvement qui leur est extérieur. Dès lors, loin de se borner à confirmer une histoire qui le précède, le texte est un facteur d'instabilité qui conteste les tendances à la stase et au monolithisme des discours synthétiques en révélant une «sismique » qui serait inhérente au champ historique. Cette démarche limitative ne peut que porter à la recherche de résultats modestes: pas question de chercher à établir une «tectonique des plaques » de la culture d'une époque, aussi restreinte soit-elle, qui permettrait de penser l'histoire des idées en termes de « rupture »; avant de revendiquer de telles 
conclusions, qui présupposent la vérification de démonstrations dont le nombre est proportionnel au niveau de généralité auquel on se place, il faut rechercher les « fêlures » qui minent toute tentative de réduction au monolithisme et qui fondent l'identité de chaque texte. Ces perturbations, fibres même de la vie de l'esprit, attendent le lecteur dans la chair du document et se révèlent au niveau microtextuel, parfois dans de simples «moments d'illogisme ou d'agrammaticalité ». D'où le besoin d'une lecture attentive qui puisse dégager l'unicité d'un texte au sein de toutes les séries périodiques ou thématiques que l'on a pu constituer autour de lui.

Cette approche nominaliste, que Terence Cave relie aux analyses de Michel de Certeau dans L'Écriture de l'histoire, part donc à la recherche des «troubles » au sein des textes, indices d'une réaction psychologique à l'apparition de phénomènes qui, s'ils sont aujourd'hui figés pour nous sous une plastique historicisée, étaient pour leurs contemporains des objets d'expérience. D'où le titre même de l'ouvrage : avant de vouloir établir des histoires continues, il est légitime de se borner à des «pré-histoires » qui interrogent les textes comme autant de «traces » d'une expérience singulière résultant d'un événement dont la perception peut être confuse; avant de déterminer généralement cet événement, il faut s'intéresser aux perceptions particulières dont il a pu être l'objet. Cette démarche permet d'articuler divers fragments d'expériences exhumés au gré des lectures afin d'établir une «topographie » des perceptions propre à une époque donnée, au sein de laquelle chaque position prend sens relativement aux autres. Si les perspectives ouvertes sont plus modestes que celles des « grands récits » historiques, elles ont l'inappréciable avantage d'être précises. Une telle réduction des ambitions de l'histoire en matière de représentations, qui va de pair avec l'intensification des exigences de celui qui l'établit et l'écrit, nous semble sous-tendue par un véritable scepticisme méthodologique, travail d'autosurveillance de la raison qui élimine de son enquête les constructions abstraites de l'esprit, en un mot dogmatiques, pour renouer avec la réalité toujours individuelle.

À partir de ces principes, Cave mène son enquête en privilégiant les zones où semble se jouer une «perturbation des systèmes d'intelligence »: l'intégration du pyrrhonisme dans le cadre intellectuel d'auteurs tels que Rabelais, Pic de La Mirandole, Henri Estienne ou Montaigne est un de ces facteurs de trouble. L'apport de Cave est ici décisif puisqu'il permet de comprendre ce que fut la réception du pyrrhonisme au-delà d'une simple question d'histoire des influences. Refusant de voir les textes s'épuiser dans un réinvestissement doctrinal, qu'il soit retour au scepticisme antique ou construction du fidéisme sceptique, l'auteur observe la prise en compte du pyrrhonisme comme expérience intellectuelle dont les ressorts psychologiques sont essentiels : folie, produit de la modernité, arme séduisante à retourner à l'intérieur de sa propre famille, ou terrifiante pour son utilisateur même, le pyrrhonisme comporte ses pathologies. Cave insiste sur la notion d'antipéristase, mouvement physique de retour par contrecoup, qui devient un schème discursif animant la plupart de ces textes mis sous la tension créée par l'irruption d'une philosophie hautement instable dans un cadre épistémologique et culturel établi : en tant que moyen dialectique d'intégration de l'inintégrable, l'antipéristase témoigne de la coexistence de réactions intellectuelles et affectives au cœur de la réception d'une simple doctrine. Autre zone sismique majeure : la question des «frontières » de la croyance qui, loin d'être fixées, subissent des déplacements discrets sous l'effet d'incursions 
sur les terres des cultes païens ou même de la sorcellerie. Selon qu'il s'agit de Ronsard, Montaigne, Bodin, Wier ou Le loyer, les syntaxes de la croyance se diversifient, chacune voyant converger des facteurs politiques, épistémologiques ou affectifs. La croyance est alors un lieu troublé par excellence, «aire de perceptions mobiles et mêmes conflictuelles » auquel on ne peut assigner un objet commun. La première partie s'achève sur l'exploration de certains aspects de la «zone médiane » du cosmos réservée aux entités intermédiaires telles que les démons, et qui réserve, pour Rabelais et Ronsard entre autres, des expériences troublantes liées à l'agonie ou à l'expérience de manifestations surnaturelles; là encore, les traces de pathos qui affleurent à la surface du texte l'éloignent d'un simple réinvestissement d'un discours du savoir. En conclusion, Cave étudie l'impact de la figure rhétorique de la paradiastole sur les éloges paradoxaux $\mathrm{du} \mathrm{XvI}^{\mathrm{e}}$ siècle : elle n'est plus comme pour Quentin Skinner un dispositif herméneutique global dont le rôle est de rendre compte de la crise de l'épistémologie à l'âge classique, mais un simple paramètre parmi d'autres qui pèse sur l'expérience et l'écriture de ces perturbations épistémologiques. La première partie de Pré-histoires, en s'attachant à observer les « fêlures » de la pensée, souligne parfaitement ce que le critique littéraire — et l'historien des idées qu'il est par force — oublie fréquemment : l'assomption d'une attitude culturelle n'est pas réductible à un objet unique qui la déterminerait; elle est affaire d'expérience singulière où les facteurs de troubles s'entrecroisent, modifiant leurs effets respectifs mais aussi leur propre réception. La seconde partie se focalise sur un problème impliqué par les questions précédentes : celui de l'émergence du «moi » que Cave explore à rebours du point d'aboutissement traditionnel qu'est Descartes. Du concept pascalien aux premières apparitions comme simple fait de langage, les « sondages » opérés autour des emplois de « moi » se doublent d'explorations au sein d'autres « outils discursifs » tels que l'allégorie, l'anamorphose et les techniques du suspens où se joue l'expression de la subjectivité. L'émergence du « je » de Philippe Desportes à Blaise Pascal s'effectuerait ainsi au gré d'un entrecroisement de diverses syntaxes liées aux champs du savoir les plus divers.

Ainsi, les innombrables analyses de détail qui charpentent ces Pré-histoires sont bien plus que des éclaircissements extrêmement précieux sur quelques auteurs majeurs; leur valeur tient justement à cette ponctualité qui empêche toute construction globale sur un auteur et toute intégration rapide au sein d'un ensemble plus englobant. Cave délivre ici la théorisation de sa propre démarche critique, réfractaire aux violences faites aux textes par l'ambition totalisante de «l'histoire (des idées) littéraire(s) » : une telle méthode, proche à notre sens de celle que revendique Montaigne pour « représenter» l'homme dans Du repentir — peinture du passage «non d'un âge en autre, mais de minute en minute » - , cherche à saisir les instants où se jouent des expériences singulières qui, même si elles sont chargées de tout un passé, irriguent la chair du texte d'un sens qui s'épuise dans une durée fragmentaire et fugace; une méthode résolument sceptique au sens antique du terme, car fondée sur la régulation du discours critique sans pour autant s'interdire tout résultat. Écrire l'histoire des idées « au seuil de la modernité » ne peut se faire alors que sous une forme proche des Essais : mettre en résonance des bribes d'expériences au lieu de les violenter au sein d'un "grand récit», les laisser entrer en écho et témoigner ainsi, sur le mode du trouble le plus souvent, de la complexité de l'humain. La 
diversification, pour ne pas dire l'entropie qui guette une telle histoire des idées, est justement le meilleur garant de son exactitude. Dès que l'historien s'adapte à la «branloire perenne » décrite par Montaigne, les «traits de [sa] peinture ne fourvoyent point » : ils cessent simplement de parler de lui, pour parler de «l'ivresse » du réel.

Emmanuel NAYA

Bram Kempers, Peinture, pouvoir et mécénat. L'essor de l'artiste professionnel dans l'Italie de la Renaissance. Trad. de l'anglais par Daniel Arasse et Catherine BÉDARD. Paris, Gérard Monfort, 1997. 17,2 × 24, 352 p., bibliogr., index.

La naissance d'un nouveau statut de l'artiste dans l'Italie de la fin du Duecento, et son développement jusqu'à l'âge baroque est l'objet de ce livre rédigé par un artistesociologue-historien d'art, enseignant à l'université d'Amsterdam. L'auteur commence par le laboratoire toscan et plus spécialement siennois. Il montre à l'œuvre dans la ville un double mécénat. Le premier est provoqué par la piété mendiante, qui gagne les élites urbaines, le second par les institutions civiques qui supervisent la décoration de la cathédrale. De Sienne, les mutations nées de ce nouveau mécénat touchent Florence. Puis papes, cardinaux et princes italiens prennent le relais et font d'Urbino, Rome ou de la Florence des Médicis les capitales artistiques des $x v^{e}$ et $x v I^{e}$ siècles. Cette synthèse beaucoup trop schématique, médiocrement illustrée - ce qui est gênant pour un tel livre —, n'a d'originalité que dans ses multiples approximations et erreurs (voir, à titre d'exemple, p. 250). C'est dommage et le sujet méritait beaucoup mieux.

Alain TALLON

Alfredo Perifano, L'Alchimie à la cour de Côme $I^{e r}$ de Médicis. Savoirs, culture et politique. Paris, Honoré Champion, 1997. $15 \times 22,5$, 251 p., bibliogr., index (Études et essais sur la Renaissance, 16).

En croisant l'étude d'une pratique et d'une réflexion scientifiques avec l'analyse d'un milieu culturel et politique - la cour de Côme ${ }^{\text {er }}$ de Médicis - Alfredo Perifano aborde d'une façon originale le statut particulier d'une «discipline de transition » : l'alchimie. L'enquête s'inscrit dans une époque, la Renaissance, où «le patrimoine intellectuel et pratique accumulé au cours des siècles dans les écrits alchimiques se prête à une utilisation assez large, au-delà des avis favorables ou contraires émis sur l'alchimie », et dans un État, la Toscane, dont le souverain, Côme $\mathrm{I}^{\mathrm{er}}$, aurait joué un véritable rôle d' « opérateur culturel » - le terme étant pris dans une triple acception de responsable, de médiateur et d'initiateur. Or, justement, 
l'alchimie, conjuguant art et science, philosophie et expérimentation, occuperait selon l'auteur une place significative dans un milieu courtisan florentin qui mêle lettrés, artistes, hommes de science et techniciens, sous le regard attentif d'un prince orientant et contrôlant formes et enjeux des débats.

Après un premier chapitre qui propose une synthèse précise et enlevée des «Aspects de l'alchimie italienne à la Renaissance », sont traités les « Aspects de l'activité scientifique à la cour de Côme $\mathrm{I}^{\mathrm{er}}{ }^{\prime}$. Ce deuxième chapitre insiste sur trois points : la fonderia (lieu clé — secret et inaccessible - d'un nouvel espace du « pouvoir»), le parcours universitaire — au studio de Pise - de Luca Ghini et, enfin, un recueil de « conseils » et de « recettes » réédité à Florence en 1550 puis en 1567 et intitulé le Ricettario fiorentino. Ce dernier marquerait tout particulièrement l'intervention du prince dans l'élaboration d'une véritable politique de santé publique. On notera d'emblée que ces trois éléments sont relativement hétérogènes et que leur appartenance à un «système » courtisan et florentin n'est pas toujours immédiatement manifeste. Le troisième et dernier chapitre du livre est consacré aux écrits sur l'alchimie rédigés par des lettrés qui sont proches de la cour (Benedetto Varchi, Sisto de Boni Sexti da Norcia, Antonio Allegretti) ou qui adressent à Côme le fruit de leur peines (Basilio Lapi, Pietro Perna et Adam de Bodestein). Ce dernier volet de l'étude s'appuie sur trois manuscrits inédits, sur un manuscrit d'Allegretti édité en 1981 par Mino Gabriele et sur une lettre de dédicace à une édition bernoise du De tartaro de Paracelse. Dans ces pages, l'auteur noue une description détaillée du contenu de «traités » souvent inconnus avec la mise en évidence, quand cela est possible, de liens personnels (ou d'affinités argumentatives) avec la cour ducale. En outre, le propos est heureusement illustré par la publication, en appendice, du texte de la lettre de dédicace au De tartaro et d'extraits de deux des manuscrits abordés précédemment. Le livre se clôt sur une importante bibliographie (qui sera d'un grand profit à quiconque veut aborder l'histoire des sciences alchimiques à la Renaissance), et sur un utile index des noms.

La thèse de l'auteur, ébauchée très brièvement dans l'avant-propos et plus longuement exposée dans la conclusion, se développe selon deux axes : d'une part, "l'alchimie en tant que savoir "transversal", science des philosophes pratiquée par les Anciens qui en avaient connu les secrets, avait un rôle à jouer dans la formation de l'image du prince »; de l'autre, «le prince alchimiste, mais en fait philosophe, devient ainsi un prince "total" s'occupant du salut de son peuple de tous les points de vue possible» (p. 149). Bref, l'alchimie serait un élément d'un complexe dispositif visant à la construction d'une image de prince sage et savant, à destination du peuple des sujets mais surtout à usage extérieur, pour affirmer dans toutes les cours d'Europe la présence et les prétentions légitimes de l'État toscan. Le propos est séduisant mais ne va pas sans soulever certaines perplexités, d'autant qu'il semble disproportionné par rapport aux conclusions partielles des trois chapitres qui précèdent.

La contribution apportée par cet ouvrage à l'histoire des sciences dans l'Italie de la Renaissance est tout à fait évidente. La présentation initiale de l'évolution de l'alchimie à la Renaissance est précise, synthétique et documentée : accessible à tout lecteur, même s'il n'est pas particulièrement féru de cette science du passé, elle permet d'entrer dans l'ouvrage sans difficulté et de mieux comprendre la fascinante aspiration à « unifier toute la nature dans un schéma cognitif et opératif unique, sans 
pour autant devenir une science intemporelle immuablement égale à elle-même » (p. 38). La recherche sur des sources manuscrites ou imprimées originales poursuivie par la suite est, quant à elle, utile aux chercheurs plus spécialisés. D'ores et déjà, la possible modification de la chronologie de l'introduction des thèses de Paracelse en Italie est, par exemple, un acquis notable de ce travail.

En revanche, dans l'étude de Perifano, la chaîne de causalité portant à la thèse forte s'il en est - que l'on a rappelée ci-dessus, s'avère moins convaincante. Le croisement, que le sous-titre de l'ouvrage annonce, entre « savoirs » ou « culture », d'un côté, et "politique », de l'autre, reste ici largement de l'ordre du postulat. Les « aspects » qui sont développés ainsi que le nombre limité et, surtout, le contenu des témoignages apportés par les sources nous donnent certes de précieux éléments de compréhension de la question de l'alchimie mais n'en constituent pas pour autant des manifestations décisives d'une place de cette même alchimie dans une "politique culturelle» de Côme I ${ }^{\mathrm{er}}$. Il nous semble que l'on en reste à une sorte de faisceau d'indices et à une série de conjectures : on pourrait d'ailleurs en voir une trace dans ces multiples passages du livre où l'auteur, avec un louable sens de la nuance, admet que plusieurs de ses assertions sont «probables", que l'on manque de sources directes (pour la fondaria notamment) ou encore que, dans tel ou tel cas, il nous présente une simple hypothèse.

L'ouvrage établit sans conteste que Côme I ${ }^{\mathrm{er}}$ manifestait un intérêt exceptionnel pour les activités scientifiques et, plus particulièrement, pour le travail sur les métaux ou les simples. Mais l'étude en a-t-elle pour autant, comme on nous l'annonçait au début, abandonné le terrain des «goûts » du prince pour celui de la « relation existant entre une situation scientifique s'inscrivant dans une période historique donnée et les initiatives [...] personnelles de l'homme d'État» (p. 43)? Peut-on vraiment, au vu des matériaux présentés ici, parler d' «initiatives » répétées, conduisant à faire de l'alchimie une des composantes d'un véritable programme global, cohérent et inscrit dans une continuité? Les quelques évocations, rappelées par Perifano, (et qui sont pour le coup très « courtisanes ») du « princemédecin » ou du «prince-philosophe » ne relèvent-elles pas pour beaucoup de topoi et de comparaisons courantes dans la pensée politique du temps? Le seul cas dans lequel nous sont fournis, à cet égard, des éléments formels concerne le ricettario puisqu'il apparaît nettement que Côme $\mathrm{I}^{\mathrm{er}}$ a bien voulu une vraie réglementation de l'activité des médecins et «pharmaciens » (et le livre nous en offre d'ailleurs d'autres exemples). Toutefois, ces pages tout à fait intéressantes nous semblent fondées en partie sur un curieux syllogisme : puisque le travail sur les simples est une des composantes de l'alchimie, et puisque le ricettario fonde ses instructions et sa pharmacopée sur les simples, le ricettario peut être intégré dans un corpus d'écrits alchimiques toscans... dont, par là même, il devient possible de dire qu'il sont inspirés, voulus et soutenus activement par l'autorité princière. Or, il faut bien dire qu'en définitive, ce lien d'interdépendance entre le travail de ces « intellectuels » ou praticiens et la «politique » du détenteur du «pouvoir » n'apparaît pas vraiment d'une grande évidence, au fil de la lecture des citations censées le mettre en évidence. Sans compter qu'il n'est pas interdit de se demander si, théoriquement, ce concept de « pouvoir », fécond il y a vingt ans, ne s'avère pas aujourd'hui trop vague et si, historiquement, dans le cas florentin, il ne tend pas à nous renvoyer l'image d'un 
gouvernement plus «absolu» et omniprésent qu'il ne l'a été ainsi que le tableau d'une sociabilité plus « courtisane » qu'elle ne le fut vraiment?

Le débat ébauché ci-dessus et les questions de méthode qu'il pose n'intéressent pas seulement l'alchimie mais touchent, plus généralement, l'analyse de la vie intellectuelle à Florence sous Côme $\mathrm{I}^{\mathrm{er}}$. L'académie florentine fut effectivement en grande partie reprise en main par l'autorité princière : ses activités connurent certes une évolution prononcée - comme le montrent les travaux de Michel Plaisance et maints lettrés firent allégeance au nouveau maître de la Toscane, dont ils sollicitèrent subsides et protection. Mais doit-on pour autant en inférer que toutes les manifestations de la vie culturelle florentine se développèrent à l'enseigne d'un contrôle strict, systématique et planifié de la part du prince en place?

La démarche suivie dans la conclusion de l'ouvrage de Perifano confirme et souligne les perplexités exposées plus haut. Cette conclusion est, en effet, fondée pour bonne part sur un long développement concernant le débat sur les éventuelles origines étrusques de la langue toscane. Or cet aspect de la «question de la langue » est justement un des points qui justifie le plus grand scepticisme sur le déploiement d'une hégémonie - très " gramscienne » - dans la Florence de Côme I ${ }^{\mathrm{er}}$. Mario Pozzi, que nous suivrons sur ce point-là (voir Mario Pozzi, « Mito aramaico-etrusco e potere assoluto a Firenze al tempo di Cosimo I ${ }^{\mathrm{er}} »$, in Le Pouvoir monarchique et ses supports idéologiques aux XIV ${ }^{e}$-XVII siècles, études réunies par Jean Dufournet, Adelin Fiorato et Augustin Redondo, Paris, Publications de la Sorbonne-Nouvelle, 1990, p. 65-76), nous semble avoir sinon réfuté du moins très largement nuancé les exégèses récentes de G. Cipriani, Sergio Bertelli et Paolo Simoncelli (sur lesquelles s'appuie le propos conclusif) : les thèses « araméennes », qui se réfèrent quasiment aux écrits des seuls Giovanbattista Gelli et Pier Francesco Giambullari, font figure d'épisode. L'unique « renfort» — explicitement demandé, et obtenu, par Gelli et Giambullari - leur fut apporté par Guillaume Postel mais celui-ci ne fit rien à l'affaire : ces élucubrations s'avérèrent ainsi rapidement de simples initiatives individuelles, souvent objets de dérision, qui ne furent jamais reprises à son compte par Côme I ${ }^{\mathrm{er}}$, malgré les efforts réels des deux auteurs précités pour que le duc fasse de leurs «étrusqueries » le fondement idéologique de son absolutisme. La politique culturelle de Côme I ${ }^{\text {er }}$ - car il y en eut une - était beaucoup moins ésotérique et sulfureuse. Elle tendait avant tout, pour fonder le prestige et les prétentions du grand-duc, à rappeler tout ce que la culture de la péninsule, mais aussi celle de l'ensemble du continent, devait au classicisme toscan (et au premier chef, à Dante, Pétrarque et Boccace), c'est-à-dire à la littérature et à la langue modernes du nouvel État.

Jean-Louis FouRnEL

Frédérique VERrIER, Les Armes de Minerve. L'humanisme militaire dans l'Italie du XVI $I^{e}$ siècle. Préf. de Christian BEC. Paris, Presses de l'université de ParisSorbonne, 1997. 15,5 × 21,5, 303 p., bibliogr., index (Jalons).

Le projet de l'ouvrage de Frédérique Verrier est de dégager à travers la figure littéraire du soldat italien $d u \mathrm{XvI}^{\mathrm{e}}$ siècle, un code de valeurs militaires alternatif au 
code chevaleresque : l'humanisme militaire. Celui-ci consiste essentiellement en une adaptation progressive de la forma mentis humaniste à la res militaris. Le culte de l'Antiquité va ainsi gagner l'armée, avec ceci de particulier à l'humanisme, que si le soldat moderne est l'héritier du fantassin romain, il est l'acteur d'une nouvelle psychomachie, où s'affrontent désormais, non plus le droit et le tort, mais la virtù et la fortuna.

Si le code chevaleresque subit des transformations, il serait faux de croire qu'il est périmé par ce nouveau code des soldats : il est démilitarisé, et va désormais régler le comportement des aristocrates en temps de paix. La matière militaire, elle, va être informée par l'humanisme.

L'armée se présente elle-même comme méritocratique : le rapport à l'argent a changé au $\mathrm{XvI}^{\mathrm{e}}$ siècle, où il devient impensable de combattre gratuitement. L'armée se professionnalise et se démocratise : la guerre est un métier, le soldat doit donc être payé. On est loin de l'idéal de désintérêt du chevalier. La guerre devient précisément le lieu où l'occasion s'offre à l'audacieux pour profiter des brusques mouvements de la fortuna. La guerre est instrumentum fortunae par excellence et, de ce fait, un garant démocratique.

Le nouveau code de valeurs fait place au mérite et à la volonté. Cette conception intellectuelle et volontariste est l'œuvre de l'humanisme et vient se substituer à l'ancienne conception aristocratique et déterministe de la guerre. Elle permet aussi d'établir que le déclin de l'Italie s'explique par un certain nombre d'erreurs qu'il est possible d'identifier et de réparer.

Parallèlement à la démocratisation de l'armée qui suit sa professionnalisation et sa spécialisation, on observe une promotion de la technique. L'art militaire se présente désormais comme un ensemble de savoirs et de techniques.

Enfin, la hiérarchisation croissante de l'armée, qui promet aux soldats une ascension sociale, achève de lui donner l'image de modèle, plus ouvert et plus mobile, de la société civile idéale.

D'autre part, se fait jour au XvI siècle la nécessité d'intellectualiser l'armée, où le soldat-lettré va devenir l'incarnation de l'humanisme militaire, rompant avec la tradition d'une armée strictement athlétique. Désormais, la force physique ne suffit plus à l'officier. Une dispute s'engage entre les armes et la plume, autour des mérites respectifs de la vie contemplative et active. Les princes eux-mêmes, surtout les illégitimes, comprennent rapidement l'avantage de la culture, qu'ils transforment en outil de propagande, en signe de distinction. Le livre se découvre un dessein sociopolitique.

La déesse Pallas-Minerve devient le symbole anthropomorphique de complémentarité des valeurs intellectuelles et militaires, même si la place du livre dans les armées reste strictement utilitariste : la lecture ne doit pas distraire mais instruire. Les soldats eux-mêmes se prennent à écrire. Mais cette écriture est cernée par l'action, passée ou future, qui reste son seul horizon avouable. Seule l'inactivité forcée permet au soldat de s'abandonner aux lettres.

Les lettres sont au cœur d'une double logique d'ouverture et de fermeture : à la fois garantes de sagesse pour les armes, et tentation d'oisiveté et de rébellion, elles sont tour à tour admirées ou repoussées. On peut observer cependant que l'assujettissement de l'art militaire à la politique fait des armes les moyens soumis aux fins que déterminent les lettres. Verrier va même plus loin en disant que les lettres et les 
armes sont paradoxalement l'âme et le bras d'un seul corps, celui de l'État, ou du prince, et qu'ainsi ce conflit des armes et des lettres rend avant tout compte des changements de la condition militaire et littéraire au $\mathrm{XvI}^{\mathrm{e}}$ siècle : elles se retrouvent à égalité, comme simples techniques que le pouvoir utilise.

Une troisième nécessité apparaît au $\mathrm{XvI}^{\mathrm{e}}$ siècle, celle d'uniformiser l'apparence des soldats. L'enjeu est aussi bien social qu'esthétique : l'indice a priori de valeur qu'était la beauté aristocratique ne suffit plus, c'est à présent la laideur du soldat qui en devient la preuve a posteriori. La mala guerra ignore les solidarités de classes ou de métiers : elle mutile les corps, défigure les soldats. L'inversion de rapports entre cavalerie et infanterie a démocratisé la profession, et vulgarisé la guerre, qui fera désormais sa place au corps envahissant du soldat, et à ses droits : désormais l'apparence est à décrypter, la valeur à reconnaître.

L'humanisme met au premier plan la formation, la valeur des qualités intellectuelles, mais aussi en relativise les critères physiques: seul un improbable puer senex, alliant l'audace et la prudence serait un soldat idéal, pour qui on ne trouve plus ni âge, ni taille idéaux. Le corps du soldat est corps en puissance, en devenir; on le pense comme projet, et non comme legs. Et cela s'applique à l'Italie tout entière : elle possède en elle-même de quoi reconquérir sa grandeur militaire.

La prudence devient la qualité essentielle du capitaine, même si l'obéissance reste celle du soldat. On voit bien aussi avec la ruralisation de la figure militaire, à quel point on a abandonné la logique aristocratique de la distinction et de l'ostentation, pour une exigence de plus grande homogénéité, d'uniformisation, qui ont une fonction autant tactique que psychologique. Il ne reste guère plus que le rôle de courtisan, à l'aristocrate qui voudrait se soustraire à ce nouveau code.

Ce qui est à remarquer, c'est que la dissociation, voire l'inversion, qu'on observe ici entre l'apparence et l'essence est strictement opposée à l'idéologie chevaleresque, qui se fondait sur une épiphanie de l'essence dans l'apparence. Cette dissociation s'inscrit dans une crise globale de l'apparence au $\mathrm{xvI}^{\mathrm{e}}$ siècle : trompeuse, il faudra s'en méfier ou l'utiliser. C'est dans ce vide, creusé entre l'essence et l'apparence, que Machiavel installera sa pensée politique.

La guerre elle-même change d'aspect : la conception chevaleresque de la guerre reposait sur l'idée de limite dans le temps, dans l'espace et dans la cruauté. Le $\mathrm{XVI}^{\mathrm{e}}$ siècle va déplacer l'enjeu moral des opérations nocturnes. On voit une approche finaliste et utilitariste de la guerre accompagner son intellectualisation, et se substituer à l'approche formaliste et moraliste de la chevalerie : l'art militaire s'émancipe de l'éthique.

Les insomnies deviennent le lot des soldats, craignant les camisades. Une analyse du statut du lit du soldat est révélatrice du discrédit qui frappe le sommeil : bien combattre, c'est à présent ne pas dormir. La nuit devient propice aux méditations, l'alternative action-inaction est remplacée par le binôme action-réflexion.

$\mathrm{Au}$ terme de sa riche et intéressante analyse, Frédérique Verrier est en mesure d'établir que le rôle de l'humanisme militaire, tant en ce qui concerne l'éthique que l'esthétique, et aussi bien comme pratique que comme théorie, fut davantage maïeutique qu'inventif. Il a contribué à donner le jour à une nouvelle idéologie militaire, laissant aux références classiques et médiévales la seule fonction d'exempla. 
Un concept subversif apparaît : le progrès. De la conciliation, on passe à la répudiation du modèle romain. Si le constat d'échec est amer pour l'Italie, il est néanmoins vide de culpabilité. Les temps ont changé, l'homme valeureux ne peut plus compter sur la fortuna.

D'un problème éthique, la guerre est devenue une question technique. On entre dans une ère de sélection où la technique brise l'unité des savoirs. Tuer devient un art, dont on parle entre spécialistes. Les diagrammes, dessins et calculs géométriques s'imposent dans les traités militaires : l'alliance des armes et des lettres a fait long feu. Dans le même temps, l'art militaire devient un hobby pour les civils : la guerre est un beau spectacle. Il revient en effet à l'art de combler l'écart entre l'être et le devoir-être, en représentant chaque chose, selon Giovanni Paolo Lomazzo, « avec l'apparence que commande la raison».

Agnès Cugno

\section{COMMERCE PHILOSOPHIQUE DU GRAND SIÈCLE}

Le Stö̈cisme au XVI et au XVII siècle. Le retour des philosophies antiques à l'âge classique. Dir. Pierre-François Moreau. Paris, Albin Michel, 1999. 14,3 × 22,5, 368 p., chronol., index (Bibliothèque Albin Michel Idées).

Ce recueil d'articles répond à un problème fondamental et épineux de l'histoire de la philosophie et de l'histoire des idées : selon quelles modalités, et à travers quels moyens d'expression, une époque vit-elle son rapport à une tradition passée? Si l'âge classique invente la modernité, il ne le fait pourtant pas sans se rapporter à l'Antiquité. Et ce rapport est irréductible à l'opposition, à la réception, à la simple utilisation. Plus que toute autre école de l'Antiquité, le stoïcisme se prête à un travail d'investigation sur ces relations qu'une époque tisse avec le passé, parce que le stoïcisme, pendant deux siècles, a imprégné de façon vivante la culture philosophique, littéraire, artistique et scientifique.

Le recueil couvre tous les champs de la culture, ce qui a pour effet de faire émerger la structuration transversale de l'époque par des problèmes posés en termes ou selon des schèmes stoïciens. Ainsi Jacques Darriulat montre, dans son étude magistrale sur la représentation de saint Sébastien dans la seconde moitié du Quattrocento, comment la virtù de Rome ressuscite en ce héros chrétien. De façon générale la grande qualité des articles réunis tient à ce que l'on ne s'est pas limité à repérer la résurgence de thèmes, de motifs, de concepts, qui seraient déposés tels quels dans le 